JOURNAL OF

FUNCTION SPACES AND APPLICATIONS

Volume 7, Number 2 (2009), 167-186 (c) 2009, Scientific Horizon

http://www.jfsa.net

\title{
Some new refinements of strengthened Hardy and Pólya-Knopp's inequalities
}

\author{
Aleksandra Čižmešija, Sabir Hussain and Josip Pečarić \\ (Communicated by Lars-Erik Persson)
}

2000 Mathematics Subject Classification. Primary 26D10, Secondary $26 \mathrm{D} 15$.

Keywords and phrases. Integral inequalities, Boas's inequality, HardyLittlewood average, Hardy's inequality, Pólya-Knopp's inequality, weights, power weights, convex functions.

Abstract. We prove a new general one-dimensional inequality for convex functions and Hardy-Littlewood averages. Furthermore, we apply this result to unify and refine the so-called Boas's inequality and the strengthened inequalities of the Hardy-Knopp-type, deriving their new refinements as special cases of the obtained general relation. In particular, we get new refinements of strengthened versions of the well-known Hardy and Pólya-Knopp's inequalities.

\section{Introduction}

To begin with, we recall some well-known classical integral inequalities. If $p>1, k \neq 1$, and the function $F$ is defined on $\mathbb{R}_{+}=\langle 0, \infty\rangle$ by

$$
F(x)= \begin{cases}\int_{0}^{x} f(t) d t, & k>1, \\ \int_{x}^{\infty} f(t) d t, & k<1,\end{cases}
$$


then the highly important Hardy's integral inequality

$$
\int_{0}^{\infty} x^{-k} F^{p}(x) d x \leq\left(\frac{p}{|k-1|}\right)^{p} \int_{0}^{\infty} x^{p-k} f^{p}(x) d x
$$

holds for all non-negative functions $f$, such that $x^{1-\frac{k}{p}} f \in L^{p}\left(\mathbb{R}_{+}\right)$. This relation was obtained by G. H. Hardy [12] in 1928, although he announced its version with $k=p>1$ already in 1920, [10], and then proved it in 1925, [11]. In [12], Hardy also pointed out that if $k$ and $F$ fulfill the conditions of the above result, but $0<p<1$, then the sign of inequality in (1.1) is reversed, that is,

$$
\int_{0}^{\infty} x^{-k} F^{p}(x) d x \geq\left(\frac{p}{|k-1|}\right)^{p} \int_{0}^{\infty} x^{p-k} f^{p}(x) d x
$$

holds. On the other hand, the first unweighted Hardy-type inequality for $p<0$ was considered by K. Knopp [20] in 1928, but in a discrete setting, for sequences of positive real numbers, while general weighted integral Hardytype inequalities for exponents $p, q<0$ and $0<p, q<1$ were first studied much later, by P. R. Beesack and H. P. Heinig [1] and H. P. Heinig [14].

Another important classical integral inequality is the so-called PólyaKnopp's inequality,

$$
\int_{0}^{\infty} \exp \left(\frac{1}{x} \int_{0}^{x} \log f(t) d t\right) d x<e \int_{0}^{\infty} f(x) d x
$$

which holds for all positive functions $f \in L^{1}\left(\mathbb{R}_{+}\right)$. This result was first published by K. Knopp [20] in 1928, but it was certainly known before since Hardy himself (see [11, p. 156]) claimed that it was G. Pólya who pointed it out to him earlier. Note that the discrete version of (1.3) is surely due to T. Carleman, [3].

It is important to observe that relations (1.1) and (1.3) are closely related since (1.3) can be obtained from (1.1) by rewriting it with the function $f$ replaced with $f^{1 / p}$ and letting $p \rightarrow \infty$. Therefore, Pólya-Knopp's inequality may be considered as a limiting case of Hardy's inequality. Moreover, the constants $\left(\frac{p}{|k-1|}\right)^{p}$ and $e$, respectively appearing on the right-hand sides of (1.1) and (1.3), are the best possible, that is, neither of them can be replaced with any smaller constant.

Since Hardy and Pólya established inequalities (1.1), (1.2), and (1.3), they have been discussed by several authors, who either gave their alternative proofs using different techniques, or applied, refined and generalized them in various ways. Further information and remarks concerning the 
rich history, development, generalizations, and applications of Hardy and Pólya-Knopp's integral inequalities can be found e.g. in the monographs $[13,22,23,25,26,27,28]$, expository papers $[6,17,21]$, and the references cited therein. Besides, here we also emphasize the papers $[2,4,5,7,8,9,18,19,24,29,32,33]$, all of which to some extent have guided us in the research we present here.

In particular, in 1970, R. P. Boas [2] proved that (1.1) and (1.3) are just special cases of a much more general inequality

$$
\int_{0}^{\infty} \Phi\left(\frac{1}{M} \int_{0}^{\infty} f(t x) d m(t)\right) \frac{d x}{x} \leq \int_{0}^{\infty} \Phi(f(x)) \frac{d x}{x}
$$

for continuous convex functions $\Phi:[0, \infty\rangle \longrightarrow \mathbb{R}$, measurable non-negative functions $f: \mathbb{R}_{+} \longrightarrow \mathbb{R}$, and non-decreasing and bounded functions $m:[0, \infty\rangle \longrightarrow \mathbb{R}$, where $M=m(\infty)-m(0)>0$ and the inner integral on the left-hand side of (1.4) is a Lebesgue-Stieltjes integral with respect to $m$. After its author, the relation (1.4) was named Boas's inequality (see also [25, Chapter IV, p. 156] and [28, Chapter 8, Theorem 8.1]). In the case of a concave function $\Phi,(1.4)$ holds with the reversed sign of inequality.

On the other hand, obviously unaware of the mentioned more general Boas's result for Hardy-Littlewood averages, in 2002, S. Kaijser et al. [18] established the so-called general Hardy-Knopp-type inequality for positive functions $f: \mathbb{R}_{+} \longrightarrow \mathbb{R}$,

$$
\int_{0}^{\infty} \Phi\left(\frac{1}{x} \int_{0}^{x} f(t) d t\right) \frac{d x}{x} \leq \int_{0}^{\infty} \Phi(f(x)) \frac{d x}{x},
$$

where $\Phi$ is a convex function on $\mathbb{R}_{+}$. By taking $\Phi(x)=x^{p}$ and $\Phi(x)=e^{x}$, they obtained an elegant new proof of inequalities (1.1) and (1.3) and showed that both Hardy and Pólya-Knopp's inequality can be derived by using only a convexity argument. Later on, A. Čižmešija et al. [9] generalized the relation (1.5) to the so-called strengthened Hardy-Knopp-type inequality by adding a weight function and truncating the range of integration to $\langle 0, b\rangle$. They also obtained a related dual inequality, that is, an inequality with the outer integrals taken over $\langle b, \infty\rangle$ and with the inner integral on the lefthand side taken over $\langle x, \infty\rangle$. These general inequalities provided an unified treatment of the strengthened Hardy and Pólya-Knopp's inequalities from $[7,8]$ and $[32,33]$.

Finally, we mention a recent paper [29] by L.-E. Persson and J. A. Oguntuase. They obtained a class of refinements of Hardy's inequality (1.1) related to an arbitrary $b \in \mathbb{R}_{+}$and the outer integrals on both hand sides of (1.1) taken over $\langle 0, b\rangle$ or $\langle b, \infty\rangle$. These results extend those of 
D. T. Shum [31] and C. O. Imoru $[15,16]$ and cover all admissible parameters $p, k \in \mathbb{R}, p \neq 0, k \neq 1$. Namely, let $f$ be a non-negative integrable function on $\langle 0, b\rangle, F(x)=\int_{0}^{x} f(t) d t$, and let $\frac{p}{k-1}>0$. If $p \in\langle-\infty, 0\rangle \cup[1, \infty\rangle$, then

$$
\int_{0}^{b} x^{-k} F^{p}(x) d x+\frac{p}{k-1} b^{1-k} F^{p}(b) \leq\left(\frac{p}{k-1}\right)^{p} \int_{0}^{b} x^{p-k} f^{p}(x) d x,
$$

while for $p \in\langle 0,1]$ inequality (1.6) holds in the reversed direction. On the contrary, if $f$ is a non-negative integrable function on $\langle b, \infty\rangle, \tilde{F}(x)=$ $\int_{x}^{\infty} f(t) d t$, and $\frac{p}{k-1}<0$, then

$$
\int_{b}^{\infty} x^{-k} \tilde{F}^{p}(x) d x+\frac{p}{1-k} b^{1-k} \tilde{F}^{p}(b) \leq\left(\frac{p}{1-k}\right)^{p} \int_{b}^{\infty} x^{p-k} f^{p}(x) d x
$$

holds for $p \in\langle-\infty, 0\rangle \cup[1, \infty\rangle$, while for $p \in\langle 0,1]$ the sign of inequality in (1.7) is reversed. The constant $\left(\frac{p}{|k-1|}\right)^{p}$ is the best possible for all cases and both inequalities (1.6) and (1.7).

Motivated by the above observations, in this paper we consider a general positive Borel measure $\lambda$ on $\mathbb{R}_{+}$, such that

$$
L=\lambda\left(\mathbb{R}_{+}\right)=\int_{0}^{\infty} d \lambda(t)<\infty,
$$

and prove a new weighted Boas-type inequality for this setting. Further, we point out that our result unifies, generalizes and refines relations (1.4) and (1.5), as well as the strengthened Hardy-Knopp-type inequalities from [9]. More precisely, applying the obtained general relation with some particular weights and a measure $\lambda$, we derive new refinements of the above inequalities. Finally, as their special cases we get new refinements of the strengthened versions of Hardy and Pólya-Knopp's inequalities, completely different from (1.6) and (1.7) and even hardly comparable with these inequalities.

The paper is organized in the following way. After this Introduction, in Section 2 we introduce some necessary notation and state, prove and discuss a general refined weighted Boas-type inequality. As its particular cases, in the same section we obtain a new refinement of inequality (1.4), as well as refinements of (1.5) and of the strengthened weighted Hardy-Knopp-type inequalities. Refinements of the strengthened Hardy and Pólya-Knopp's inequalities are presented in the concluding Section 3 of the paper, along with some final remarks.

Conventions. Throughout this paper, all measures are assumed to be positive, all functions are assumed to be measurable, and expressions of the 
form $0 \cdot \infty, \frac{0}{0}, \frac{a}{\infty}(a \in \mathbb{R})$, and $\frac{\infty}{\infty}$ are taken to be equal to zero. As usual, by $d x$ we denote the Lebesgue measure on $\mathbb{R}$, by a weight function (shortly: a weight) we mean a non-negative measurable function on the actual interval, while an interval in $\mathbb{R}$ is any convex subset of $\mathbb{R}$. Finally, by Int $I$ we denote the interior of an interval $I \subseteq \mathbb{R}$.

\section{The main results}

First, we introduce some necessary notation and, for reader's convenience, recall some basic facts about convex functions. Let $I$ be an interval in $\mathbb{R}$ and $\Phi: I \longrightarrow \mathbb{R}$ be a convex function. For $x \in I$, by $\partial \Phi(x)$ we denote the subdifferential of $\Phi$ at $x$, that is, the set $\partial \Phi(x)=\{\alpha \in \mathbb{R}$ : $\Phi(y)-\Phi(x)-\alpha(y-x) \geq 0, y \in I\}$. It is well-known that $\partial \Phi(x) \neq \emptyset$ for all $x \in \operatorname{Int} I$. More precisely, at each point $x \in \operatorname{Int} I$ we have $-\infty<\Phi_{-}^{\prime}(x) \leq \Phi_{+}^{\prime}(x)<\infty$ and $\partial \Phi(x)=\left[\Phi_{-}^{\prime}(x), \Phi_{+}^{\prime}(x)\right]$, while the set on which $\Phi$ is not differentiable is at most countable. Moreover, every function $\varphi: I \longrightarrow \mathbb{R}$ for which $\varphi(x) \in \partial \Phi(x)$, whenever $x \in \operatorname{Int} I$, is increasing on Int $I$. For more details about convex functions see e.g. a recent monograph [26].

On the other hand, for a finite Borel measure $\lambda$ on $\mathbb{R}_{+}$, that is, having property (1.8), and a Borel measurable function $f: \mathbb{R}_{+} \longrightarrow \mathbb{R}$, by $A f$ we denote its Hardy-Littlewood average, defined in terms of the Lebesgue integral as

$$
A f(x)=\frac{1}{L} \int_{0}^{\infty} f(t x) d \lambda(t), \quad x \in \mathbb{R}_{+},
$$

where $L$ is defined by (1.8).

Now, we can state and prove the main result of this paper. It is given in the following theorem.

Theorem 2.1. Let $\lambda$ be a finite Borel measure on $\mathbb{R}_{+}, L$ be defined by (1.8), and let $u$ and $v$ be non-negative measurable functions on $\mathbb{R}_{+}$, where

$$
v(x)=\int_{0}^{\infty} u\left(\frac{x}{t}\right) d \lambda(t)<\infty, \quad x \in \mathbb{R}_{+} .
$$

Let $\Phi$ be a continuous convex function on an interval $I \subseteq \mathbb{R}$ and $\varphi: I \longrightarrow \mathbb{R}$ be any function, such that $\varphi(x) \in \partial \Phi(x)$ for all $x \in \operatorname{Int} I$. If $f: \mathbb{R}_{+} \longrightarrow \mathbb{R}$ is a measurable function, such that $f(x) \in I$ for all $x \in \mathbb{R}_{+}$, and $A f(x)$ is defined by (2.1), then $A f(x) \in I$, for all $x \in \mathbb{R}_{+}$, and the inequality 


$$
\begin{aligned}
& \frac{1}{L} \int_{0}^{\infty} v(x) \Phi(f(x)) \frac{d x}{x}-\int_{0}^{\infty} u(x) \Phi(A f(x)) \frac{d x}{x} \\
& \geq \frac{1}{L}\left|\int_{0}^{\infty} \int_{0}^{\infty} u(x)\right| \Phi(f(t x))-\Phi(A f(x)) \mid d \lambda(t) \frac{d x}{x} \\
& \quad-\int_{0}^{\infty} \int_{0}^{\infty} u(x)|\varphi(A f(x))||f(t x)-A f(x)| d \lambda(t) \frac{d x}{x} \mid
\end{aligned}
$$

holds.

Proof. $\quad$ For a fixed $x \in \mathbb{R}_{+}$, let $h_{x}: \mathbb{R}_{+} \longrightarrow \mathbb{R}$ be defined by $h_{x}(t)=f(t x)-A f(x)$. Then (1.8) and (2.1) imply

$$
\int_{0}^{\infty} h_{x}(t) d \lambda(t)=\int_{0}^{\infty} f(t x) d \lambda(t)-A f(x) \int_{0}^{\infty} d \lambda(t)=0 .
$$

Now, suppose $x \in \mathbb{R}_{+}$is such that $A f(x) \notin I$. Observing that $f\left(\mathbb{R}_{+}\right) \subseteq I$ and that $I$ is an interval in $\mathbb{R}$, we have $h_{x}(t)>0$ for all $t \in \mathbb{R}_{+}$, or $h_{x}(t)<0$ for all $t \in \mathbb{R}_{+}$, that is, the function $h_{x}$ is either strictly positive or strictly negative. Since this contradicts (2.4), we have proved that $A f(x) \in I$, for all $x \in \mathbb{R}_{+}$. Note that if $A f(x)$ is an endpoint of $I$ for some $x \in \mathbb{R}_{+}$(in cases when $I$ is not an open interval), then $h_{x}\left(\right.$ or $\left.-h_{x}\right)$ will be a nonnegative function whose integral over $\mathbb{R}_{+}$, with respect to the measure $\lambda$, is equal to 0 . Therefore, $h_{x} \equiv 0$, that is, $f(t x)=A f(x)$ holds for $\lambda$ a.e. $t \in \mathbb{R}_{+}$. To prove inequality (2.3), observe that for all $r \in \operatorname{Int} I$ and $s \in I$ we have

$$
\Phi(s)-\Phi(r)-\varphi(r)(s-r) \geq 0,
$$

where $\varphi: I \longrightarrow \mathbb{R}$ is any function such that $\varphi(x) \in \partial \Phi(x)$ for $x \in \operatorname{Int} I$, and hence

$$
\begin{aligned}
\Phi(s)-\Phi(r)-\varphi(r)(s-r) & =|\Phi(s)-\Phi(r)-\varphi(r)(s-r)| \\
& \geq|| \Phi(s)-\Phi(r)|-| \varphi(r)|| s-r \| .
\end{aligned}
$$

Especially, in the case when $A f(x) \in \operatorname{Int} I$, by substituting $r=A f(x)$ and $s=f(t x)$ in $(2.5)$, for all $t \in \mathbb{R}_{+}$we get

$$
\begin{aligned}
& \Phi(f(t x))-\Phi(A f(x))-\varphi(A f(x))[f(t x)-A f(x)] \\
& (2.6) \geq \geq|| \Phi(f(t x))-\Phi(A f(x))|-| \varphi(A f(x))|| f(t x)-A f(x) \| .
\end{aligned}
$$

On the other hand, the above analysis provides (2.6) to hold even if $A f(x)$ is an endpoint of $I$, since in that case both sides of inequality (2.6) are equal to 0 for $\lambda$-a.e. $t \in \mathbb{R}_{+}$. Multiplying (2.6) by $\frac{u(x)}{x}$, then integrating it 
over $\mathbb{R}_{+}^{2}$ with respect to the measures $d \lambda(t)$ and $\frac{d x}{x}$, and applying Fubini's theorem, we obtain the following sequence of inequalities:

$$
\begin{aligned}
\int_{0}^{\infty} \int_{0}^{\infty} u(x) \Phi(f(t x)) d \lambda(t) \frac{d x}{x}-\int_{0}^{\infty} \int_{0}^{\infty} u(x) \Phi(A f(x)) d \lambda(t) \frac{d x}{x} \\
\quad-\int_{0}^{\infty} \int_{0}^{\infty} u(x) \varphi(A f(x))[f(t x)-A f(x)] d \lambda(t) \frac{d x}{x} \\
\geq \quad \int_{0}^{\infty} \int_{0}^{\infty} u(x)|| \Phi(f(t x))-\Phi(A f(x)) \mid \\
\quad-|\varphi(A f(x))||f(t x)-A f(x)| \mid d \lambda(t) \frac{d x}{x} \\
\geq \quad \int_{0}^{\infty} u(x)\left|\int_{0}^{\infty}\right| \Phi(f(t x))-\Phi(A f(x)) \mid d \lambda(t) \\
\quad-|\varphi(A f(x))| \int_{0}^{\infty}|f(t x)-A f(x)| d \lambda(t) \mid \frac{d x}{x} \\
\geq\left|\int_{0}^{\infty} \int_{0}^{\infty} u(x)\right| \Phi(f(t x))-\Phi(A f(x)) \mid d \lambda(t) \frac{d x}{x} \\
\quad-\int_{0}^{\infty} \int_{0}^{\infty} u(x)|\varphi(A f(x))||f(t x)-A f(x)| d \lambda(t) \frac{d x}{x} \mid .
\end{aligned}
$$

Again, by using Fubini's theorem and the substitution $y=t x$, the first integral on the left-hand side of (2.7) becomes

$$
\begin{aligned}
\int_{0}^{\infty} & \int_{0}^{\infty} u(x) \Phi(f(t x)) d \lambda(t) \frac{d x}{x} \\
= & \int_{0}^{\infty} \int_{0}^{\infty} u(x) \Phi(f(t x)) \frac{d x}{x} d \lambda(t) \\
= & \int_{0}^{\infty} \int_{0}^{\infty} u\left(\frac{y}{t}\right) \Phi(f(y)) \frac{d y}{y} d \lambda(t) \\
= & \int_{0}^{\infty} \Phi(f(y)) \int_{0}^{\infty} u\left(\frac{y}{t}\right) d \lambda(t) \frac{d y}{y} \\
= & \int_{0}^{\infty} v(y) \Phi(f(y)) \frac{d y}{y},
\end{aligned}
$$

while for the second integral we have

$$
\int_{0}^{\infty} \int_{0}^{\infty} u(x) \Phi(A f(x)) d \lambda(t) \frac{d x}{x}=L \int_{0}^{\infty} u(x) \Phi(A f(x)) \frac{d x}{x} .
$$


174 Some new refinements of Hardy and Pólya-Knopp's inequalities

Finally, considering (2.4), we similarly get

$$
\begin{aligned}
\int_{0}^{\infty} & \int_{0}^{\infty} u(x) \varphi(A f(x))[f(t x)-A f(x)] d \lambda(t) \frac{d x}{x} \\
& =\int_{0}^{\infty} u(x) \varphi(A f(x))\left(\int_{0}^{\infty} h_{x}(t) d \lambda(t)\right) \frac{d x}{x}=0
\end{aligned}
$$

so (2.3) holds by combining (2.7), (2.8), (2.9), and (2.10).

Remark 2.1. Observe that (2.7) provides a pair of inequalities interpolated between the left-hand side and the right-hand side of (2.3), that is, further new refinements of (2.3).

Remark 2.2. If $\Phi$ is a concave function (that is, if $-\Phi$ is convex), then (2.5) reads

$$
\begin{aligned}
\Phi(r)-\Phi(s)-\varphi(r)(r-s) & =|\Phi(r)-\Phi(s)-\varphi(r)(r-s)| \\
& \geq|| \Phi(s)-\Phi(r)|-| \varphi(r)|| s-r \|,
\end{aligned}
$$

where $\varphi$ is a real function on $I$ such that $\varphi(x) \in \partial \Phi(x)=\left[\Phi_{+}^{\prime}(x), \Phi_{-}^{\prime}(x)\right]$, for all $x \in \operatorname{Int} I$. Therefore, in this setting (2.3) holds by its left-hand side replaced with

$$
\int_{0}^{\infty} u(x) \Phi(A f(x)) \frac{d x}{x}-\frac{1}{L} \int_{0}^{\infty} v(x) \Phi(f(x)) \frac{d x}{x} .
$$

Moreover, if $\Phi$ is an affine function, then (2.3) becomes equality.

Since the right-hand side of (2.3) is non-negative, as an immediate consequence of Theorem 2.1 and Remark 2.2 we get the following result, a weighted Boas's inequality.

Corollary 2.1. Suppose $\lambda$ is a finite Borel measure on $\mathbb{R}_{+}, L$ is defined by (1.8), $u$ is a non-negative measurable function on $\mathbb{R}_{+}$, and the function $v$ is defined on $\mathbb{R}_{+}$by (2.2). If $\Phi$ is a continuous convex function on an interval $I \subseteq \mathbb{R}$, then the inequality

$$
\int_{0}^{\infty} u(x) \Phi(A f(x)) \frac{d x}{x} \leq \frac{1}{L} \int_{0}^{\infty} v(x) \Phi(f(x)) \frac{d x}{x}
$$

holds for all measurable functions $f: \mathbb{R}_{+} \longrightarrow \mathbb{R}$, such that $f(x) \in I$ for all $x \in \mathbb{R}_{+}$, where $A f(x)$ is defined by (2.1). For a concave function $\Phi$, the sign of inequality in relation (2.11) is reversed.

In the sequel, we analyze some important particular cases of Theorem 2.1 and Corollary 2.1 and compare them with some results previously known 
from the literature. Namely, by setting $u(x) \equiv 1$, we obtain a refined Boas-type inequality with $A f(x)$ defined by the Lebesgue integral.

Corollary 2.2. Let $\lambda$ be a finite Borel measure on $\mathbb{R}_{+}$and $L$ be defined by (1.8). Then the inequality

$$
\begin{aligned}
\int_{0}^{\infty} \Phi(f(x)) & \frac{d x}{x}-\int_{0}^{\infty} \Phi(A f(x)) \frac{d x}{x} \\
\geq & \frac{1}{L}\left|\int_{0}^{\infty} \int_{0}^{\infty}\right| \Phi(f(t x))-\Phi(A f(x)) \mid d \lambda(t) \frac{d x}{x} \\
& -\int_{0}^{\infty} \int_{0}^{\infty}|\varphi(A f(x))||f(t x)-A f(x)| d \lambda(t) \frac{d x}{x} \mid
\end{aligned}
$$

holds for all continuous convex functions $\Phi$ on an interval $I \subseteq \mathbb{R}$, real functions $\varphi$ on $I$, such that $\varphi(x) \in \partial \Phi(x)$ for $x \in \operatorname{Int} I$, and all measurable real functions $f$ on $\mathbb{R}_{+}$, such that $f(x) \in I$ for all $x \in \mathbb{R}_{+}$, and $A f(x)$ defined by (2.1). If the function $\Phi$ is concave, then (2.1) holds with

$$
\int_{0}^{\infty} \Phi(A f(x)) \frac{d x}{x}-\int_{0}^{\infty} \Phi(f(x)) \frac{d x}{x}
$$

on its left-hand side.

Evidently, Corollary 2.2 implies the following analogue of (1.4).

Corollary 2.3. If $\lambda$ is a finite Borel measure on $\mathbb{R}_{+}, L$ is defined by (1.8), $\Phi$ is a continuous convex function on an interval $I \subseteq \mathbb{R}, f$ is a measurable real function on $\mathbb{R}_{+}$with values in $I$, and $A f(x)$ is defined by (2.1), then

$$
\int_{0}^{\infty} \Phi(A f(x)) \frac{d x}{x} \leq \int_{0}^{\infty} \Phi(f(x)) \frac{d x}{x} .
$$

If $\Phi$ is concave, then the sign of inequality in (2.13) is reversed.

Remark 2.3. Let $m:[0, \infty\rangle \longrightarrow \mathbb{R}$ be a non-decreasing bounded function and $M=m(\infty)-m(0)>0$. It is well-known that $m$ induces a finite Borel measure $\lambda$ on $\mathbb{R}_{+}$(and vice versa), such that the related Lebesgue and Lebesgue-Stieltjes integrals are equivalent. Thus, all the above results from this section can be interpreted as for $A f(x)$ defined by the Lebesgue-Stieltjes integral with respect to $m$, that is, as

$$
A f(x)=\frac{1}{M} \int_{0}^{\infty} f(t x) d m(t), \quad x \in \mathbb{R}_{+} .
$$

Therefore, our results refine and generalize Boas's inequality (1.4). Namely, we obtained a refinement of its weighted version. 
176 Some new refinements of Hardy and Pólya-Knopp's inequalities

To conclude this section, we consider measures $\lambda$ which yield refinements of the Hardy-Knopp-type inequalities mentioned in the Introduction. Especially, for $d \lambda(t)=\chi_{[0,1]}(t) d t$ we obtain a refinement of a weighted version of (1.5).

Theorem 2.2. Let $u$ be a non-negative function on $\mathbb{R}_{+}$, such that the function $t \mapsto \frac{u(t)}{t^{2}}$ is locally integrable in $\mathbb{R}_{+}$, and let

$$
w(x)=x \int_{x}^{\infty} u(t) \frac{d t}{t^{2}}, \quad t \in \mathbb{R}_{+} .
$$

If a real-valued function $\Phi$ is convex on an interval $I \subseteq \mathbb{R}$ and $\varphi: I \longrightarrow \mathbb{R}$ is such that $\varphi(x) \in \partial \Phi(x)$ for all $x \in \operatorname{Int} I$, then the inequality

$$
\begin{aligned}
& \int_{0}^{\infty} w(x) \Phi(f(x)) \frac{d x}{x}-\int_{0}^{\infty} u(x) \Phi(H f(x)) \frac{d x}{x} \\
& \geq\left|\int_{0}^{\infty} u(x) \int_{0}^{x}\right| \Phi(f(t))-\Phi(H f(x)) \mid d t \frac{d x}{x^{2}} \\
&-\int_{0}^{\infty} u(x)|\varphi(H f(x))| \int_{0}^{x}|f(t)-H f(x)| d t \frac{d x}{x^{2}} \mid
\end{aligned}
$$

holds for all functions $f$ on $\mathbb{R}_{+}$with values in $I$ and for $H f(x)$ defined by

$$
H f(x)=\frac{1}{x} \int_{0}^{x} f(t) d t
$$

for $x \in \mathbb{R}_{+}$. If $\Phi$ is a concave function, then (2.14) holds with

$$
\int_{0}^{\infty} u(x) \Phi(H f(x)) \frac{d x}{x}-\int_{0}^{\infty} w(x) \Phi(f(x)) \frac{d x}{x}
$$

on its left-hand side.

Proof. Follows directly from Theorem 2.1 and Remark 2.2, rewritten with the measure $d \lambda(t)=\chi_{[0,1]}(t) d t$. In this setting, we have $L=1$,

$A f(x)=\int_{0}^{1} f(t x) d t=H f(x)$ and $v(x)=\int_{0}^{1} u\left(\frac{x}{t}\right) d t=w(x), \quad x \in \mathbb{R}_{+}$,

so (2.14) holds.

Remark 2.4. Let a convex function $\Phi$ and functions $u, w, f$, and $H f$ be as in Theorem 2.2. Observing that the right-hand side of relation (2.14) 
is non-negative, we get

$$
\int_{0}^{\infty} u(x) \Phi(H f(x)) \frac{d x}{x} \leq \int_{0}^{\infty} w(x) \Phi(f(x)) \frac{d x}{x} .
$$

Moreover, for a concave function $\Phi$ relation (2.16) holds with the reversed sign of inequality. This result, the so-called weighted Hardy-Knopp-type inequality, was already obtained in [9, Theorem 1], while its particular case (1.5), originally proved in [18], follows by setting $u(x) \equiv 1$. Therefore, $(2.14)$ may be regarded as a refined weighted inequality of the Hardy-Knopp type and relation (2.3) as its generalization.

On the other hand, a dual result to Theorem 2.2 can be derived by considering $(2.3)$ with $d \lambda(t)=\chi_{[1, \infty\rangle}(t) \frac{d t}{t^{2}}$.

Theorem 2.3. Suppose $u: \mathbb{R}_{+} \longrightarrow \mathbb{R}$ is a non-negative function, locally integrable in $\mathbb{R}_{+}$, and $w$ is defined on $\mathbb{R}_{+}$by

$$
w(x)=\frac{1}{x} \int_{0}^{x} u(t) d t
$$

If $\Phi$ is a convex function on an interval $I \subseteq \mathbb{R}$ and $\varphi: I \longrightarrow \mathbb{R}$ is such that $\varphi(x) \in \partial \Phi(x)$ for all $x \in \operatorname{Int} I$, then the inequality

$$
\begin{aligned}
& \int_{0}^{\infty} w(x) \Phi(f(x)) \frac{d x}{x}-\int_{0}^{\infty} u(x) \Phi(\tilde{H} f(x)) \frac{d x}{x} \\
& \geq\left|\int_{0}^{\infty} u(x) \int_{x}^{\infty}\right| \Phi(f(t))-\Phi(\tilde{H} f(x)) \mid \frac{d t}{t^{2}} d x \\
& \quad-\int_{0}^{\infty} u(x)|\varphi(\tilde{H} f(x))| \int_{x}^{\infty}|f(t)-\tilde{H} f(x)| \frac{d t}{t^{2}} d x \mid
\end{aligned}
$$

holds for all functions $f$ on $\mathbb{R}_{+}$with values in $I$ and for $\tilde{H} f(x)$ defined by

$$
\tilde{H} f(x)=x \int_{x}^{\infty} f(t) \frac{d t}{t^{2}}
$$

for $x \in \mathbb{R}_{+}$. In the case when $\Phi$ is concave, (2.17) holds if its left-hand side is replaced with

$$
\int_{0}^{\infty} u(x) \Phi(\tilde{H} f(x)) \frac{d x}{x}-\int_{0}^{\infty} w(x) \Phi(f(x)) \frac{d x}{x} .
$$

Proof. Set $d \lambda(t)=\chi_{[1, \infty\rangle}(t) \frac{d t}{t^{2}}$ in Theorem 2.1 and Remark 2.2. Then

$$
A f(x)=\int_{1}^{\infty} f(t x) \frac{d t}{t^{2}}=\tilde{H} f(x), \quad v(x)=\int_{1}^{\infty} u\left(\frac{x}{t}\right) \frac{d t}{t^{2}}=w(x), \quad x \in \mathbb{R}_{+},
$$


178 Some new refinements of Hardy and Pólya-Knopp's inequalities

and $L=1$, so $(2.17)$ holds.

Remark 2.5. As in Remark 2.4, note that for a convex function $\Phi$ and functions $u, w, f$, and $\tilde{H} f$ from the statement of Theorem 2.3, we have

$$
\int_{0}^{\infty} u(x) \Phi(\tilde{H} f(x)) \frac{d x}{x} \leq \int_{0}^{\infty} w(x) \Phi(f(x)) \frac{d x}{x},
$$

while for a concave $\Phi$ relation (2.19) holds with the inequality sign $\geq$. Since as a consequence of Theorem 2.1 and Theorem 2.3 we derived a dual inequality to (2.16), relation (2.17) can be considered as a refined dual weighted Hardy-Knopp-type inequality and (2.3) as its generalization.

Finally, as special cases of Theorem 2.2 and Theorem 2.3, we formulate refinements of the strengthened Hardy-Knopp-type inequalities.

Corollary 2.4. Suppose $b \in \mathbb{R}_{+}, u:\langle 0, b\rangle \longrightarrow \mathbb{R}$ is a non-negative function, such that the function $t \mapsto \frac{u(t)}{t^{2}}$ is locally integrable in $\langle 0, b\rangle$, and the function $w$ is defined by

$$
w(x)=x \int_{x}^{b} u(t) \frac{d t}{t^{2}}, \quad x \in\langle 0, b\rangle .
$$

If $\Phi$ is a convex function on an interval $I \subseteq \mathbb{R}$ and $\varphi: I \longrightarrow \mathbb{R}$ is such that $\varphi(x) \in \partial \Phi(x)$ for all $x \in \operatorname{Int} I$, then the inequality

$$
\begin{aligned}
& \int_{0}^{b} w(x) \Phi(f(x)) \frac{d x}{x}-\int_{0}^{b} u(x) \Phi(H f(x)) \frac{d x}{x} \\
& \geq\left|\int_{0}^{b} u(x) \int_{0}^{x}\right| \Phi(f(t))-\Phi(H f(x)) \mid d t \frac{d x}{x^{2}} \\
&-\int_{0}^{b} u(x)|\varphi(H f(x))| \int_{0}^{x}|f(t)-H f(x)| d t \frac{d x}{x^{2}} \mid
\end{aligned}
$$

holds for all functions $f:\langle 0, b\rangle \longrightarrow \mathbb{R}$ with values in $I$ and $H f$ defined on $\langle 0, b\rangle$ by (2.15). If $\Phi$ is a concave function, the order of integrals on the left-hand side of (2.20) is reversed.

Proof. Let $\hat{u}, \hat{w}$, and $\hat{f}$ be defined on $\mathbb{R}_{+}$by $\hat{u}(x)=u(x) \chi_{\langle 0, b\rangle}(x)$,

$$
\hat{w}(x)=x \int_{x}^{\infty} \hat{u}(t) \frac{d t}{t^{2}}=w(x) \chi_{\langle 0, b\rangle}(x),
$$

and $\hat{f}(x)=f(x) \chi_{\langle 0, b\rangle}(x)+c \chi_{[b, \infty\rangle}(x)$, where $c \in I$ is arbitrary. Since these functions naturally extend $u, w$, and $f$ to act on $\mathbb{R}_{+}$, they evidently fulfill 
the conditions of Theorem 2.2, considered with $\hat{u}, \hat{w}$, and $\hat{f}$ instead of $u$, $w$, and $f$ respectively. Therefore, (2.14) holds and in this setting it becomes $(2.20)$.

Remark 2.6. Since the right-hand side of (2.20) is non-negative, Corollary 2.4 improves a result from [9, Theorem 1]. Hence, it can be considered as a refined strengthened Hardy-Knopp-type inequality.

Remark 2.7. For $u(x) \equiv 1$, we have $w(x)=1-\frac{x}{b}$ in Corollary 2.4, so (2.20) reads

$$
\begin{aligned}
\int_{0}^{b}\left(1-\frac{x}{b}\right) & \Phi(f(x)) \frac{d x}{x}-\int_{0}^{b} \Phi(H f(x)) \frac{d x}{x} \\
\geq & \left|\int_{0}^{b} \int_{0}^{x}\right| \Phi(f(t))-\Phi(H f(x)) \mid d t \frac{d x}{x^{2}} \\
& -\int_{0}^{b}|\varphi(H f(x))| \int_{0}^{x}|f(t)-H f(x)| d t \frac{d x}{x^{2}} \mid .
\end{aligned}
$$

This relation provides a basis for results in the following section.

A dual result to inequality (2.20) is given in the next corollary.

Corollary 2.5. For $b \in \mathbb{R}, b \geq 0$, let $u:\langle b, \infty\rangle \longrightarrow \mathbb{R}$ be a non-negative locally integrable function in $\langle b, \infty\rangle$ and the function $w$ be given by

$$
w(x)=\frac{1}{x} \int_{b}^{x} u(t) d t, \quad x \in\langle b, \infty\rangle .
$$

Let $\Phi$ be a convex function on an interval $I \subseteq \mathbb{R}$ and $\varphi: I \longrightarrow \mathbb{R}$ be such that $\varphi(x) \in \partial \Phi(x)$ for all $x \in \operatorname{Int} I$. Then the inequality

$$
\begin{aligned}
& \int_{b}^{\infty} w(x) \Phi(f(x)) \frac{d x}{x}-\int_{b}^{\infty} u(x) \Phi(\tilde{H} f(x)) \frac{d x}{x} \\
& \geq\left|\int_{b}^{\infty} u(x) \int_{x}^{\infty}\right| \Phi(f(t))-\Phi(\tilde{H} f(x)) \mid \frac{d t}{t^{2}} d x \\
&-\int_{b}^{\infty} u(x)|\varphi(\tilde{H} f(x))| \int_{x}^{\infty}|f(t)-\tilde{H} f(x)| \frac{d t}{t^{2}} d x \mid
\end{aligned}
$$

holds for all functions $f$ on $\langle b, \infty\rangle$ with values in $I$ and $\tilde{H} f$ defined by (2.18). For a concave function $\Phi$, the order of integrals on the left-hand side of (2.22) is reversed.

Proof. As in the proof of Corollary 2.4, inequality (2.22) follows by applying Theorem 2.3 to the functions $\hat{u}, \hat{w}$, and $\hat{f}$, where $\hat{u}(x)=$ 
$u(x) \chi_{\langle b, \infty\rangle}(x)$

$$
\hat{w}(x)=\frac{1}{x} \int_{0}^{x} \hat{u}(t) d t=w(x) \chi_{\langle b, \infty\rangle}(x),
$$

and $\hat{f}(x)=c \chi_{\langle 0, b]}(x)+f(x) \chi_{\langle b, \infty\rangle}(x)$, for an arbitrary $c \in I$.

Remark 2.8. Note that (2.22) refines [9, Theorem 2] since the right-hand side of (2.22) is non-negative. Thus, we obtained a refined strengthened dual Hardy-Knopp-type inequality.

Remark 2.9. For $u(x) \equiv 1,(2.22)$ reads

$$
\begin{aligned}
\int_{b}^{\infty}\left(1-\frac{b}{x}\right) & \Phi(f(x)) \frac{d x}{x}-\int_{b}^{\infty} \Phi(\tilde{H} f(x)) \frac{d x}{x} \\
\geq & \left|\int_{b}^{\infty} \int_{x}^{\infty}\right| \Phi(f(t))-\Phi(\tilde{H} f(x)) \mid \frac{d t}{t^{2}} d x \\
& -\int_{b}^{\infty}|\varphi(\tilde{H} f(x))| \int_{x}^{\infty}|f(t)-\tilde{H} f(x)| \frac{d t}{t^{2}} d x \mid .
\end{aligned}
$$

Together with (2.21), we shall use this inequality to derive refinements of the classical Hardy and Pólya-Knopp's inequalities.

\section{Refinements of strengthened Hardy and Pólya-Knopp's inequalities}

In the previous section, obtained inequalities were discussed with respect to a measure $\lambda$ and a weight function $u$, while a convex function $\Phi$ remained unspecified. On the contrary, here we consider two particular convex (or concave) functions, namely $\Phi(x)=x^{p}$ and $\Phi(x)=e^{x}$, and derive some new refinements of the well-known Hardy and Pólya-Knopp's inequalities, as well as their strengthened versions. Moreover, we show that they are just special cases of the results mentioned.

We start with new refinements of Hardy's inequality, so let $p \in \mathbb{R}, p \neq 0$, and $\Phi(x)=x^{p}$. Obviously, $\varphi(x)=\Phi^{\prime}(x)=p x^{p-1}, x \in \mathbb{R}_{+}$, and the function $\Phi$ is convex for $p \in \mathbb{R} \backslash[0,1\rangle$, concave for $p \in\langle 0,1]$, and affine for $p=1$. On the other hand, for a locally integrable function $f: \mathbb{R}_{+} \longrightarrow \mathbb{R}$, as in the Introduction, we denote

$$
F(x)=\int_{0}^{x} f(t) d t \quad \text { and } \quad \tilde{F}(x)=\int_{x}^{\infty} f(t) d t, \quad x \in \mathbb{R}_{+} .
$$


A new refined strengthened Hardy's inequality is given in the following corollary.

Corollary 3.1. Let $0<b \leq \infty$ and $p, k \in \mathbb{R}$ be such that $p \neq 0$, $k \neq 1$, and $\frac{p}{k-1}>0$. Let $f$ be a non-negative function on $\langle 0, b\rangle$. If $p \in\langle-\infty, 0\rangle \cup[1, \infty\rangle$, then the inequality

$$
\begin{aligned}
& \left(\frac{p}{k-1}\right)^{p} \int_{0}^{b}\left[1-\left(\frac{x}{b}\right)^{\frac{k-1}{p}}\right] x^{p-k} f^{p}(x) d x-\int_{0}^{b} x^{-k} F^{p}(x) d x \\
& \geq \mid\left(\frac{p}{k-1}\right)^{p-1} \int_{0}^{b} x^{\frac{1-k}{p}-1} \int_{0}^{x} t^{\frac{k-1}{p}-1} \\
& \quad \times \quad t^{p-k+1} f^{p}(t)-\left(\frac{k-1}{p}\right)^{p} x^{1-k} F^{p}(x) \mid d t d x \\
& \quad-|p| \int_{0}^{b} x^{-k} F^{p-1}(x) \int_{0}^{x}\left|f(t)-\frac{k-1}{p} \cdot \frac{1}{t}\left(\frac{t}{x}\right)^{\frac{k-1}{p}} F(x)\right| d t d x \mid
\end{aligned}
$$

holds. In the case when $p \in\langle 0,1\rangle$, the order of integrals of the right-hand side of inequality (3.2) is reversed.

Proof. First, let either $p \geq 1, k>1$, or $p<0, k<1$, and let $\Phi(x)=x^{p}$ and $\varphi(x)=p x^{p-1}$. According to Corollary 2.4 and Remark 2.7, then (2.21) holds. Rewriting it for $a=b^{\frac{k-1}{p}}$ and $x \mapsto f\left(x^{\frac{p}{k-1}}\right) x^{\frac{p}{k-1}-1}$, instead of $b$ and $f$ respectively, we get

$$
\begin{gathered}
\int_{0}^{a}\left(1-\frac{x}{a}\right) x^{p\left(\frac{p}{k-1}-1\right)} f^{p}\left(x^{\frac{p}{k-1}}\right) \frac{d x}{x}-\int_{0}^{a}\left(\frac{1}{x} \int_{0}^{x} f\left(t^{\frac{p}{k-1}}\right) t^{\frac{p}{k-1}-1} d t\right)^{p} \frac{d x}{x} \\
\geq\left|\int_{0}^{a} \int_{0}^{x}\right| t^{p\left(\frac{p}{k-1}-1\right)} f^{p}\left(t^{\frac{p}{k-1}}\right)-\left(\frac{1}{x} \int_{0}^{x} f\left(r^{\frac{p}{k-1}}\right) r^{\frac{p}{k-1}-1} d r\right)^{p} \mid d t \frac{d x}{x^{2}} \\
-\int_{0}^{a}\left|p\left(\frac{1}{x} \int_{0}^{x} f\left(r^{\frac{p}{k-1}}\right) r^{\frac{p}{k-1}-1} d r\right)^{p-1}\right| \\
\times \int_{0}^{x}\left|f\left(t^{\frac{p}{k-1}}\right) t^{\frac{p}{k-1}-1}-\frac{1}{x} \int_{0}^{x} f\left(r^{\frac{p}{k-1}}\right) r^{\frac{p}{k-1}-1} d r\right| d t \frac{d x}{x^{2}} \mid
\end{gathered}
$$

so (3.2) follows by a sequence of substitutions such as $s=x^{\frac{p}{k-1}}$. The remaining case, that is, when $p \in\langle 0,1\rangle$ and $k>1$, is a direct consequence of Corollary 2.4 and Remark 2.7.

Now, we state and prove a refined strengthened dual Hardy's inequality. 
182 Some new refinements of Hardy and Pólya-Knopp's inequalities

Corollary 3.2. Suppose $0 \leq b<\infty$ and $p, k \in \mathbb{R}$ are such that $p \neq 0$, $k \neq 1$, and $\frac{p}{k-1}<0$. If $p \in\langle-\infty, 0\rangle \cup[1, \infty\rangle$, then the inequality

$$
\begin{aligned}
& \left(\frac{p}{1-k}\right)^{p} \int_{b}^{\infty}\left[1-\left(\frac{b}{x}\right)^{\frac{1-k}{p}}\right] x^{p-k} f^{p}(x) d x-\int_{b}^{\infty} x^{-k} \tilde{F}^{p}(x) d x \\
& \geq \mid\left(\frac{p}{1-k}\right)^{p-1} \int_{b}^{\infty} x^{\frac{1-k}{p}-1} \\
& \quad \times \quad \int_{x}^{\infty} t^{\frac{k-1}{p}-1}\left|t^{p-k+1} f^{p}(t)-\left(\frac{1-k}{p}\right)^{p} x^{1-k} \tilde{F}^{p}(x)\right| d t d x \\
& \quad-|p| \int_{b}^{\infty} x^{-k} \tilde{F}^{p-1}(x) \int_{x}^{\infty}\left|f(t)-\frac{1-k}{p} \cdot \frac{1}{t}\left(\frac{x}{t}\right)^{\frac{1-k}{p}} \tilde{F}(x)\right| d t d x \mid
\end{aligned}
$$

holds for all non-negative functions $f$ on $\langle b, \infty\rangle$. In the case when $p \in\langle 0,1\rangle$, the order of integrals on the left-hand side of inequality (3.3) is reversed.

Proof. As in the proof of Corollary 3.1, we use $\Phi(x)=x^{p}$, that is, $\varphi(x)=p x^{p-1}$, and rewrite inequality (2.23) for $b$ and $f$ respectively replaced with $a=b^{\frac{1-k}{p}}$ and $x \mapsto f\left(x^{\frac{p}{1-k}}\right) x^{\frac{p}{1-k}+1}$. Relation (3.3) then follows by a sequence of substitutions of the form $s=x^{\frac{p}{1-k}}$. Note that we again distinguish two cases. The first one, which yields (3.3), holds when either $p \geq 1, k<1$, or $p<0, k>1$. In the other one, with $p \in\langle 0,1\rangle$ and $k<1$, the order of integrals on the left-hand side of inequality (3.3) is reversed.

Remark 3.1. Observe that for $b=\infty$ the left-hand side of (3.2) reads

$$
\left(\frac{p}{k-1}\right)^{p} \int_{0}^{\infty} x^{p-k} f^{p}(x) d x-\int_{0}^{\infty} x^{-k} F^{p}(x) d x
$$

while for $b=0$ on the left-hand side of (3.3) we have

$$
\left(\frac{p}{1-k}\right)^{p} \int_{0}^{\infty} x^{p-k} f^{p}(x) d x-\int_{0}^{\infty} x^{-k} \tilde{F}^{p}(x) d x .
$$

Therefore, we obtained a refinement of the classical Hardy's inequality (1.1). Also note that for $p=1$ relations (3.2) and (3.2) are trivial since their both sides are equal to 0 .

Remark 3.2. Observe that Corollary 3.1 and Corollary 3.2 provide new and original refinements of Hardy's inequality although the idea to strengthen and refine (1.1) is not new and results of such type already exist in the literature. As in the Introduction, here we just mention the papers 
$[15,16],[31]$, and a recent paper [29]. It is important to emphasize that our results are completely different from those given in these papers and even hardly comparable with (1.6) and (1.7). A third type of refinements of a similar form can be found in another recent paper [30], where a fairly new concept of superquadratic function was used in a crucial way.

Finally, we consider $\Phi(x)=e^{x}$ to obtain refinements of the strengthened Pólya-Knopp's inequality and of its dual. For a positive function $f$ on $\mathbb{R}_{+}$ and $x \in \mathbb{R}_{+}$, we denote

$G f(x)=\exp \left(\frac{1}{x} \int_{0}^{x} \log f(t) d t\right) \quad$ and $\quad \tilde{G} f(x)=\exp \left(x \int_{x}^{\infty} \log f(t) \frac{d t}{t^{2}}\right)$.

Related results are given in the following two corollaries.

Corollary 3.3. Let $0<b \leq \infty$ and $f$ be a positive function on $\langle 0, b\rangle$. Then

$$
\begin{aligned}
e \int_{0}^{b}\left(1-\frac{x}{b}\right) & f(x) d x-\int_{0}^{b} G f(x) d x \\
\geq & \left|\int_{0}^{b} \int_{0}^{x}\right| e t f(t)-x G f(x) \mid d t \frac{d x}{x^{2}} \\
& -\int_{0}^{b} G f(x) \int_{0}^{x}\left|\log \frac{e t f(t)}{x G f(x)}\right| d t \frac{d x}{x} \mid .
\end{aligned}
$$

Corollary 3.4. If $0 \leq b<\infty$ and $f$ is a positive function on $\langle b, \infty\rangle$, then

$$
\begin{aligned}
& \frac{1}{e} \int_{b}^{\infty}\left(1-\frac{b}{x}\right) f(x) d x-\int_{b}^{\infty} \tilde{G} f(x) d x \\
& \geq\left|\int_{b}^{\infty} \int_{x}^{\infty}\right| \frac{1}{e} t f(t)-x \tilde{G} f(x) \mid \frac{d t}{t^{2}} d x \\
& \quad-\int_{b}^{\infty} x \tilde{G} f(x) \int_{x}^{\infty}\left|\log \frac{t f(t)}{\operatorname{ex} \tilde{G} f(x)}\right| \frac{d t}{t^{2}} d x \mid
\end{aligned}
$$

Remark 3.3. Note that for $b=\infty$ in (3.4) we have a refined PólyaKnopp's inequality, while for $b=0$ relation (3.5) becomes its refined dual inequality.

Acknowledgements. The research of the first and the third author was supported by the Croatian Ministry of Science, Education and Sports, under the Research Grants 058-1170889-1050 (first author) and 117-1170889-0888 (third author). The last two authors also acknowledge with thanks the facilities provided to them by the Abdus Salam School of Mathematical 
184 Some new refinements of Hardy and Pólya-Knopp's inequalities

Sciences, GC University, Lahore, Pakistan. We thank the careful referee and Professor Lars-Erik Persson for valuable comments and suggestions, which we have used to improve the final version of this paper.

\section{References}

[1] P.R. Beesack and H.P. Heinig, Hardy's inequalities with indices less than 1, Proc. Amer. Math. Soc., 83 (1981), 532-536.

[2] R.P. Boas, Some integral inequalities related to Hardy's inequality, J. Anal. Math., 23 (1970), 53-63.

[3] T. Carleman, Sur les fonctions quasi-analytiques, Comptes rendus du $\mathrm{V}^{\mathrm{e}}$ Congres des Mathematiciens Scandinaves, Helsingfors 1922, 181196.

[4] J.A. Cochran and C.S. Lee, Inequalities related to Hardy's and Heinig's, Math. Proc. Cambridge Philos. Soc., 96 (1984), 1-7.

[5] A. Čižmešija and J. Pečarić, Mixed means and Hardy's inequality, Math. Inequal. Appl., 1 (1998), 491-506.

[6] A. Čižmešija and J. Pečarić, Classical Hardy's and Carleman's inequalities and mixed means, in: T. M. Rassias (ed.), Survey on Classical Inequalities, Kluwer Academic Publishers, Dordrecht/Boston/London, 2000, 27-65.

[7] A. Čižmešija and J. Pečarić, Some new generalisations of inequalities of Hardy and Levin-Cochran-Lee, Bull. Austral. Math. Soc., 63 (2001), 105-113.

[8] A. Čižmešija and J. Pečarić, On Bicheng-Debnath's generalizations of Hardy's integral inequality, Int. J. Math. Math. Sci., 27 (2001), 237250.

[9] A. Čižmešija, J. Pečarić, and L.-E. Persson, On strengthened Hardy and Pólya-Knopp's inequalities, J. Approx. Theory, 125 (2003), 74-84.

[10] G.H. Hardy, Note on a theorem of Hilbert, Math. Z., 6 (1920), 314-317.

[11] G.H. Hardy, Notes on some points in the integral calculus LX: An inequality between integrals (60), Messenger of Math., 54 (1925), 150156.

[12] G.H. Hardy, Notes on some points in the integral calculus LXIV, Messenger of Math., 57 (1928), 12-16.

[13] G.H. Hardy, J. E. Littlewood, and G. Pólya, Inequalities, 2nd edition, Cambridge University Press, Cambridge, 1967. 
[14] H.P. Heinig, Variations af Hardy's inequality, Real Anal. Exchange, 5 (1979-80), 61-81.

[15] C.O. Imoru, On some integral inequalities related to Hardy's, Canad. Math. Bull., 20 (1977), 307-312.

[16] C.O. Imoru, On some extensions of Hardy's inequality, Int. J. Math. Math. Sci., 8 (1985), 165-171.

[17] M. Johansson, L.-E. Persson, and A. Wedestig, Carleman's inequality: history, proofs and some new generalizations, J. Inequal. Pure Appl. Math., 3 (2003), 19 pages, electronic.

[18] S. Kaijser, L.-E. Persson, and A. Öberg, On Carleman and Knopp's Inequalities, J. Approx. Theory, 117 (2002), 140-151.

[19] S. Kaijser, L. Nikolova, L-E. Persson, and A. Wedestig, Hardy type inequalities via convexity, Math. Inequal. Appl., 8 (2005), 403-417.

[20] K. Knopp, Über Reihen mit positiven Gliedern, J. London Math. Soc., 3 (1928), 205-211.

[21] A. Kufner, L. Maligranda, and L.-E. Persson, The prehistory of the Hardy inequality, Amer. Math. Monthly, 113 (2006), 715-732.

[22] A. Kufner, L. Maligranda, and L.-E. Persson, The Hardy Inequality - About its History and Some Related Results, Vydavatelsky Servis Publishing House, Pilsen, 2007.

[23] A. Kufner and L.-E. Persson, Weighted Inequalities of Hardy Type, World Scientific Publishing Co, Singapore/ New Jersey/ London/Hong Kong, 2003.

[24] E.R. Love, Inequalities related to those of Hardy and of Cochran and Lee, Math. Proc. Cambridge Philos. Soc., 99 (1986), 395-408.

[25] D.S. Mitrinović, J. E. Pečarić, and A. M. Fink, Inequalities involving functions and their integrals and derivatives, Kluwer Academic Publishers, Dordrecht/Boston/London, 1991.

[26] C. Niculescu and L.-E. Persson, Convex Functions and Their Applications. A Contemporary Approach, CMC Books in Mathematics, Springer, New York, 2006.

[27] B. Opic and A. Kufner, Hardy-Type Inequalities, Pitman Research Notes in Mathematics Series, Longman Scientific \& Technical, Harlow, 1990.

[28] J.E. Pečarić, F. Proschan, and Y. L. Tong, Convex Functions, Partial Orderings, and Statistical Applications, Academic Press, San Diego, 1992. 
186 Some new refinements of Hardy and Pólya-Knopp's inequalities

[29] L.-E. Persson and J.A. Oguntuase, Refinement of Hardy's inequality for "all" p, in Banach and Function Spaces II, eds. M. Kato and L. Maligranda, Yokohama Publishers (Proceedings of the Second International Symposium on Banach and Function Spaces, Kitakyushu, Japan, 2006), pp. 129-144, to appear (2008).

[30] J.A. Oguntuase and L.-E. Persson, Refinement of Hardy's inequalities via superquadratic and subquadratic functions, J. Math. Anal. Appl., 339 (2008), 1305-1312.

[31] D.T. Shum, On integral inequalities related to Hardy's, Canad. Math. Bull., 14 (1971), 225-230.

[32] B. Yang and L. Debnath, Generalizations of Hardy's integral inequalities, Internat. J. Math. \& Math. Sci., 22 (1999), 535-542.

[33] B. Yang, Z. Zeng, and L. Debnath, On new generalizations of Hardy's integral inequality, J. Math. Anal. Appl., 217 (1998), 321-327.

Department of Mathematics

University of Zagreb

Bijenička cesta 30, 10000 Zagreb

Croatia

(E-mail : cizmesij@math.hr)

Abdus Salam School of Mathematical Sciences

GC University

35-C-II, M. M. Alam Road, Gulberg III, Lahore - 54660

Pakistan

(E-mail : sabirhus@gmail.com)

Faculty of Textile Technology

University of Zagreb

Pierottijeva 6, 10000 Zagreb

Croatia

(E-mail : pecaric@element.hr)

(Received : April 2008) 


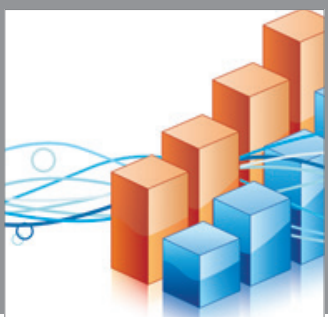

Advances in

Operations Research

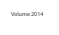

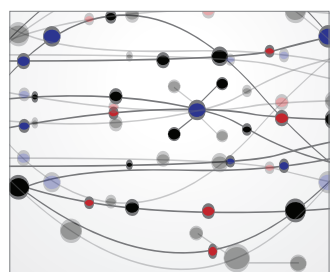

\section{The Scientific} World Journal
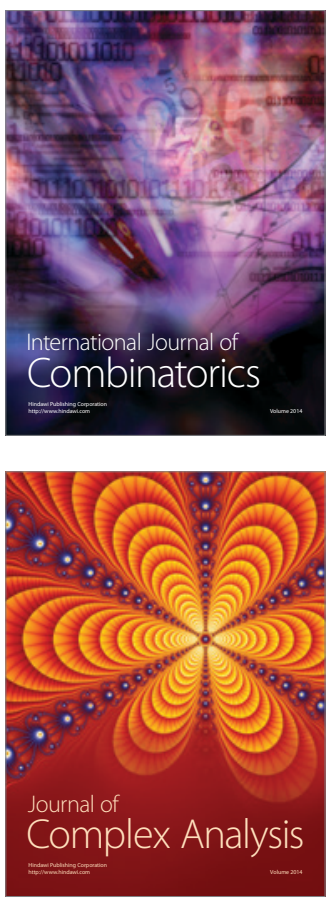

International Journal of

Mathematics and

Mathematical

Sciences
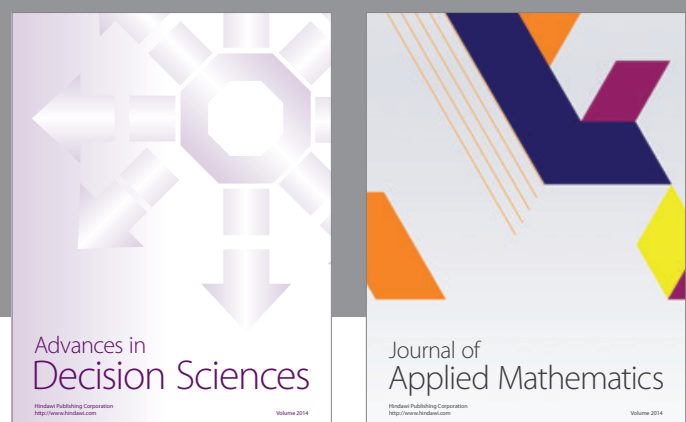

Journal of

Applied Mathematics
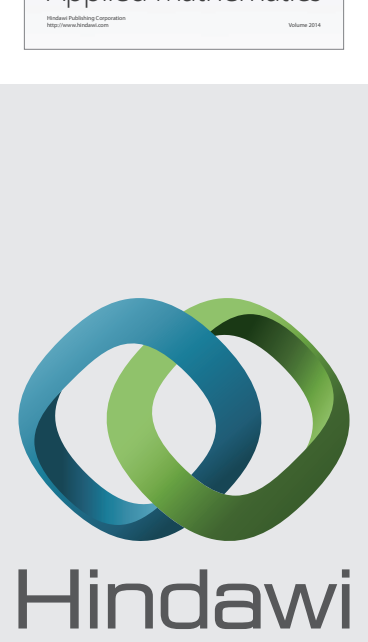

Submit your manuscripts at http://www.hindawi.com
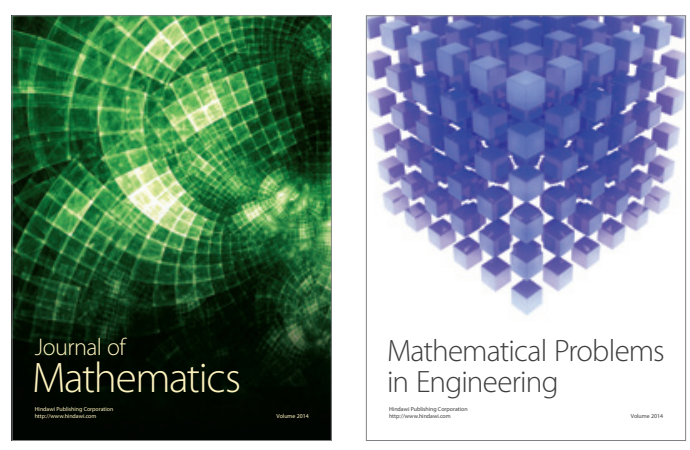

Mathematical Problems in Engineering
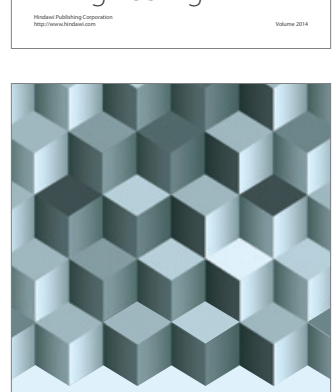

Journal of

Function Spaces
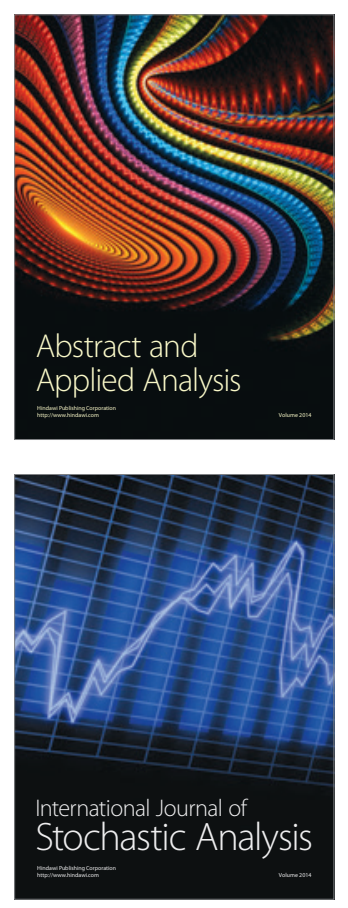

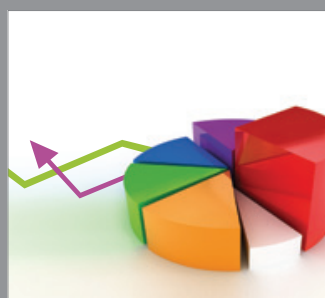

ournal of

Probability and Statistics

Promensencen
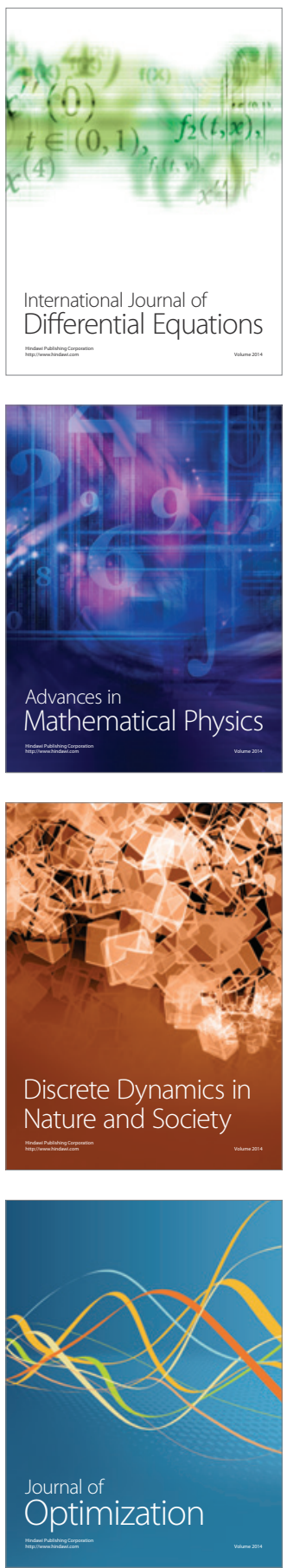